\title{
Transitive sensitive subsystems for interval maps
}

\author{
by \\ Sylvie Ruette (Paris)
}

\begin{abstract}
We prove that for continuous interval maps the existence of a non-empty closed invariant subset which is transitive and sensitive to initial conditions is implied by positive topological entropy and implies chaos in the sense of $\mathrm{Li}-$ Yorke, and we exhibit examples showing that these three notions are distinct.
\end{abstract}

1. Introduction. In this paper an interval map is a topological dynamical system given by a continuous map $f: I \rightarrow I$ where $I$ is a compact interval. In the literature much has been said about chaos for interval maps. The point is that the relations between various properties related to chaos are much more numerous for these systems than for general dynamical systems. As a consequence there is a rather ordered "scale of chaos" on the interval. For example, for interval maps topological weak mixing and topological strong mixing are equivalent [3], and transitivity implies sensitivity to initial conditions [2], which in turn implies positive topological entropy [8]. For more details on this topic see e.g. [6], [15, §§6-9] and [21].

Among the different definitions of chaos, a well known one is chaos in the sense of $L i-$ Yorke. The definition follows the ideas of [17] but was formalized later.

Definition 1.1. Let $T: X \rightarrow X$ be a continuous map on the metric space $X$ with distance $d$. Then $T$ is called chaotic in the sense of Li-Yorke if there exists an uncountable set $S \subset X$ such that, for all distinct $x, y \in S$,

$$
\limsup _{n \rightarrow+\infty} d\left(T^{n}(x), T^{n}(y)\right)>0 \text { and } \liminf _{n \rightarrow+\infty} d\left(T^{n}(x), T^{n}(y)\right)=0 .
$$

Note that in the definition of chaos in the sense of Li-Yorke some people make the extra assumption that for all $x \in S$ and all periodic points $z \in X$ one has $\lim \sup _{n \rightarrow+\infty} d\left(T^{n}(x), T^{n}(z)\right)>0$. This gives an equivalent definition since this property is satisfied by all points of the set $S$ except at most one [6, p. 144].

2000 Mathematics Subject Classification: Primary 37E05.

The author has been partly supported by a Marie Curie Fellowship of the European Community programme Human Potential under contract $n^{\circ}$ HPMF-CT-2002-02026. 
$\mathrm{Li}$ and Yorke showed that an interval map with a periodic point of period 3 is chaotic in the sense of Li-Yorke [17]. In [14] Janková and Smítal generalized this result as follows:

Theorem 1.2 (Janková-Smítal). If $f: I \rightarrow I$ is an interval map of positive entropy, then it is chaotic in the sense of Li-Yorke.

Recently, Blanchard, Glasner, Kolyada and Maass [4] proved that, if $T: X \rightarrow X$ is a continuous map on the compact metric space $X$ such that the topological entropy of $T$ is positive, then the system is chaotic in the sense of Li-Yorke.

The converse of this result is not true, even for interval maps: Smítal [23] and Xiong [25] built interval maps of zero entropy which are chaotic in the sense of Li-Yorke. See also [20] (a correction is given in [18]) or [11] for examples of $C^{\infty}$ interval maps which are chaotic in the sense of Li-Yorke and have a null entropy.

Recall that the map $T: X \rightarrow X$ is transitive if for all non-empty open subsets $U, V$ there exists an integer $n \geq 0$ such that $T^{-n}(U) \cap V \neq \emptyset$; if $X$ is compact with no isolated point, then $T$ is transitive if and only if there exists $x \in X$ such that $\omega(x, T)=X$ (where $\omega(x, T)$ is the set of limit points of $\left.\left\{T^{n}(x) \mid n \geq 0\right\}\right)$. The map $T$ has sensitive dependence on initial conditions (or simply is sensitive) if there exists $\delta>0$ such that for all $x \in X$ and all neighbourhoods $U$ of $x$ there exist $y \in U$ and $n \geq 0$ such that $d\left(T^{n}(x), T^{n}(y)\right) \geq \delta$. A subset $Y \subset X$ is invariant if $T(Y) \subset Y$.

The work of Wiggins [24] leads to the following definition (see, e.g., [13]).

Definition 1.3. Let $X$ be a metric space. The continuous map $T: X$ $\rightarrow X$ is said to be chaotic in the sense of Wiggins if there exists a nonempty closed invariant subset $Y$ such that the restriction $\left.T\right|_{Y}$ is transitive and sensitive.

The aim of this paper is to locate this notion with respect to the other definitions of chaos.

REmark 1.4. A continuous map $T: X \rightarrow X$ which is transitive and sensitive is sometimes called chaotic in the sense of Auslander-Yorke [1]. If in addition the periodic points are dense, then it is called chaotic in the sense of Devaney [10].

Transitive sensitive subsystems appear naturally when considering a horseshoe, that is, two disjoint closed intervals $J, K$ such that $f(J) \cap f(K) \supset$ $J \cup K$, because the points whose orbits never escape from $J \cup K$ form a subset on which $f$ acts almost like a 2 -shift [5]. For interval maps, positive entropy is equivalent to the existence of a horseshoe for some power of $f[19,7]$ (see also [6, Chap. VIII]), and one can deduce that a positive entropy interval 
map has a transitive, sensitive subsystem. More precisely, Shihai Li proved the following result [16].

Theorem 1.5 (Shihai Li). Let $f: I \rightarrow I$ be an interval map. The topological entropy of $f$ is positive if and only if there exists a non-empty closed invariant subset $X \subset I$ such that $\left.f\right|_{X}$ is transitive, sensitive to initial conditions, and the periodic points are dense in $X$ (in other words, $\left.f\right|_{X}$ is Devaney chaotic).

In the "if" part of this theorem one cannot suppress the assumption on the periodic points. In Section 3 we build a counter-example which leads to the following theorem.

TheOREM 1.6. There exists a continuous map $f:[0,1] \rightarrow[0,1]$ of zero topological entropy which is chaotic in the sense of Wiggins.

In [23] Smítal built a zero entropy map $f$ which is chaotic in the sense of $\mathrm{Li}-$ Yorke. If one looks at the construction of $f$, it is not hard to prove that $\left.f\right|_{\omega(0, f)}$ is transitive and sensitive to initial conditions. We show the following theorem in Section 2.

TheOREM 1.7. Let $f: I \rightarrow I$ be an interval map. If $f$ is Wiggins chaotic then it is Li-Yorke chaotic.

The converse of this theorem is not true, contrary to what one may expect by considering Smítal's example. The last and longest section is devoted to the construction of a counter-example that proves the following result.

THEOREM 1.8. There exists a continuous interval map $g: I \rightarrow I$ which is chaotic in the sense of Li-Yorke but not in the sense of Wiggins.

From Theorems 1.5 to 1.8 it follows that, for interval maps, chaos in the sense of Wiggins is a strictly intermediate notion between positive entropy and chaos in the sense of $\mathrm{Li}-$ Yorke.

Furthermore the examples of Sections 3 and 4 show that the behaviour of zero entropy interval maps is more varied that one might expect. Let us describe the different kinds of dynamics exhibited by these maps.

The next result is well known (see, e.g., [6, p. 218]).

Theorem 1.9. Let $f: I \rightarrow I$ be an interval map. The following properties are equivalent:

- the topological entropy of $f$ is zero,

- every periodic point has period $2^{n}$ for some integer $n \geq 0$.

According to Sharkovski1's Theorem [22] the set of periods of periodic points of a zero entropy interval map is either $\left\{2^{k} ; 0 \leq k \leq n\right\}$ for some 
integer $n$, and then $f$ is said to be of type $2^{n}$, or $\left\{2^{k} ; k \geq 0\right\}$, and then $f$ is of type $2^{\infty}$. There is little to say about the dynamics of type $2^{n}$, and some interval maps of type $2^{\infty}$ share almost the same dynamics [9]: every orbit converges to some periodic orbit of period $2^{k}$; these maps are never Li-Yorke chaotic.

The interval maps of type $2^{\infty}$ that admit an infinite $\omega$-limit set may be Li-Yorke chaotic or not, as shown by Smítal [23]. A map $f$ that is not LiYorke chaotic is called "uniformly non-chaotic" in [6] and it has the following property: every point $x$ is approximately periodic, that is, for every $\varepsilon>0$ there exists a periodic point $y$ and an integer $N$ such that $\mid f^{n}(x)-f^{n}(y)[<\varepsilon$ for all $n \geq N$.

The maps built in Sections 3 and 4 are both zero entropy and Li-Yorke chaotic. In the first example there is a transitive sensitive subsystem which is the core of the dynamics; in particular Li-Yorke chaos can be read on this subsystem. In the second example this situation does not occur since there is no transitive sensitive subsystem.

2. Wiggins chaos implies $\mathbf{L i}-$ Yorke chaos. The following notion of $f$-non-separable points was introduced by Smítal to give an equivalent condition for chaos in the sense of Li-Yorke [23]. Note that Theorem 2.2 was proven to remain valid for all interval maps by Janková and Smítal [14].

Definition 2.1. Let $f: I \rightarrow I$ be an interval map and $a_{0}, a_{1}$ two distinct points in $I$. The points $a_{0}, a_{1}$ are called $f$-separable if there exist two disjoint subintervals $J_{0}, J_{1}$ and two integers $n_{0}, n_{1}$ such that for $i=0,1, a_{i} \in J_{i}$, $f^{n_{i}}\left(J_{i}\right)=J_{i}$ and $\left(f^{k}\left(J_{i}\right)\right)_{0 \leq k<n_{i}}$ are disjoint. Otherwise they are $f$-nonseparable.

Theorem 2.2 (Smítal). Let $f: I \rightarrow I$ be an interval map of zero entropy. The following properties are equivalent:

- $f$ is chaotic in the sense of Li-Yorke,

- there exists $x_{0} \in I$ such that the set $\omega\left(x_{0}, f\right)$ is infinite and contains two f-non-separable points.

In the proof of this theorem, Smital showed the following intermediate result which describes the structure of an infinite $\omega$-limit set of a zero entropy map.

Lemma 2.3. Let $f: I \rightarrow I$ be an interval map of zero entropy and $x_{0}$ in $I$ such that $\omega\left(x_{0}, f\right)$ is infinite. For all $n \geq 0$ and $0 \leq i<2^{n}$, define

$$
I_{n}^{i}=\left[\min \omega\left(f^{i}\left(x_{0}\right), f^{2^{n}}\right), \max \omega\left(f^{i}\left(x_{0}\right), f^{2^{n}}\right)\right], \quad L_{n}^{i}=\bigcup_{k \geq 0} f^{k 2^{n}}\left(I_{n}^{i}\right) .
$$


Then $f\left(L_{k}^{i}\right)=L_{k}^{i+1 \bmod 2^{k}}$ for all $0 \leq i<2^{k}$, and the intervals $\left(L_{k}^{i}\right)_{0 \leq i<2^{k}}$ are pairwise disjoint.

Lemma 2.4. Let $f:[a, b] \rightarrow \mathbb{R}$ be a continuous map. If $f([a, b]) \supset[a, b]$, then $f$ has a fixed point.

Proof. There exist $x, y \in[a, b]$ such that $f(x) \leq a$ and $f(y) \geq b$. One then has $f(x)-x \leq a-x \leq 0$ and $f(y)-y \geq b-y \geq 0$, so there is a point $c \in[x, y]$ such that $f(c)-c=0$.

Lemma 2.5. Let $f: I \rightarrow I$ be an interval map of zero entropy. If $J \subset I$ is $a$ (not necessarily closed) subinterval such that $f^{p}(J)=J$ and $\left(f^{i}(J)\right)_{0 \leq i<p}$ are pairwise disjoint then $p$ is a power of 2 .

Proof. If $J$ is reduced to one point then it is a periodic orbit and by Theorem 1.9, $p$ is a power of 2 . We assume that $J$ is non-degenerate, which implies that $f^{n}(J)$ is a non-degenerate interval for all $n \geq 0$.

Since $f^{p}(\bar{J})=\bar{J}$, by Lemma 2.4 there exists $x \in \bar{J}$ such that $f^{p}(x)=x$. According to Theorem 1.9 the period of $x$ is equal to $2^{k}$ for some $k$; write $p=m 2^{k}$. If $x \in J$ then $\left(f^{i}(x)\right)_{0 \leq i<p}$ are distinct and $p=2^{k}$.

Suppose that $m \geq 3$. Then $x \in \partial J$; we assume that $x=\sup J$, the case of $x=\inf J$ being symmetric. Since $x=f^{2^{k}}(x) \in f^{2^{k}}(\bar{J})$ and $f^{2^{k}}(J) \cap J=\emptyset$, one has $x=\inf f^{2^{k}}(J)$. But also $x \in f^{2^{k+1}}(\bar{J})$, which contradicts the fact that $J, f^{2^{k}}(J), f^{2^{k+1}}(J)$ are pairwise disjoint non-degenerate intervals. Therefore $m=1$ or 2 and $p$ is a power of 2 .

The following result is the key tool in the proof of Theorem 1.7. A rather similar result can be found in a paper of Fedorenko, Sharkovskiu and Smítal [12].

Lemma 2.6. Let $f: I \rightarrow I$ be an interval map of zero entropy and $x_{0}$ in I such that $\omega\left(x_{0}, f\right)$ is infinite and does not contain two $f$-non-separable points. Then for all $\varepsilon>0$ there exists $\delta>0$ such that if $x, y \in \omega\left(x_{0}, f\right)$ and $|x-y|<\delta$, then $\left|f^{n}(x)-f^{n}(y)\right|<\varepsilon$ for all $n \geq 0$.

Proof. Let $X=\omega\left(x_{0}, f\right)$. For all integers $n \geq 0$ and $0 \leq i<2^{n}$ define $a_{n}^{i}=\min \omega\left(f^{i}\left(x_{0}\right), f^{2^{n}}\right)$ and $b_{n}^{i}=\max \omega\left(f^{i}\left(x_{0}\right), f^{2^{n}}\right)$. Define $I_{n}^{i}$ and $L_{n}^{i}$ as in Lemma 2.3; then $I_{n}^{i}=\left[a_{n}^{i}, b_{n}^{i}\right]$. The points $a_{n}^{i}, b_{n}^{i}$ belong to $X$ and

$$
I_{n+1}^{i} \cup I_{n+1}^{i+2^{n}} \subset I_{n}^{i} \quad \text { for all } 0 \leq i<2^{n} .
$$

Suppose that there exists $\varepsilon>0$ such that

$$
\text { for all } n \geq 0 \text { there is } 0 \leq i<2^{n} \text { with }\left|I_{n}^{i}\right| \geq \varepsilon \text {. }
$$

Using (1) we can build a sequence $\left(i_{n}\right)_{n \geq 0}$ such that $I_{n+1}^{i_{n+1}} \subset I_{n}^{i_{n}}$ and $\left|I_{n}^{i_{n}}\right| \geq \varepsilon$ for all $n \geq 0$. Define $I_{\infty}=\bigcap_{n \geq 1} I_{n}^{i_{n}}$. It is a decreasing intersection of compact intervals, hence a closed interval, and $\left|I_{\infty}\right| \geq \varepsilon$. Write $I_{\infty}=[a, b]$; then 


$$
a=\lim _{n \rightarrow+\infty} a_{n}^{i_{n}}, \quad b=\lim _{n \rightarrow+\infty} b_{n}^{i_{n}},
$$

and thus $a, b \in X$. One has $a, b \in L_{n}^{i_{n}}$ for all $n \geq 0$. By Lemma 2.3 the intervals $L_{n+2}^{i_{n+2}}, f^{2^{n}}\left(L_{n+2}^{i_{n+2}}\right), f^{2^{n+1}}\left(L_{n+2}^{i_{n+2}}\right)$ are pairwise disjoint, so $\left\{a, f^{2^{n}}(a)\right.$, $\left.f^{2^{n+1}}(a)\right\}$ are distinct.

Since $a \neq b$ and by assumption $a, b$ are $f$-separable, there exist an interval $J$ and an integer $p \geq 1$ such that $a \in J, b \notin J, f^{p}(J)=J$ and $\left(f^{i}(J)\right)_{0 \leq i<p}$ are pairwise disjoint. By Lemma 2.5, $p$ is a power of 2 ; write $p=2^{k}$.

Consider the interval $K=L_{k}^{i_{k}} \cap J$; it contains $a$, and $f^{2^{k}}(K) \subset K$ because $f^{2^{k}}\left(L_{k}^{i_{k}}\right)=L_{k}^{i_{k}}$ by Lemma 2.3. Thus $K$ contains $\left\{a, f^{2^{k}}(a), f^{2^{k+1}}(a)\right\}$. These three points belong to $\omega\left(x_{0}, f\right)$ and are distinct, so one of them belongs to $\operatorname{Int}(K)$ and there exists an integer $n$ such that $f^{n}\left(x_{0}\right) \in K$. We thus have

$$
X=\omega\left(x_{0}, f\right) \subset \bigcup_{j=0}^{2^{k}-1} f^{j}(\bar{K}) .
$$

Let $b^{\prime} \in X$ be such that $f^{2^{k+1}}\left(b^{\prime}\right)=b$ and let $0 \leq j<2^{k}$ be such that $b^{\prime} \in f^{j}(\bar{K})$. The points $b^{\prime}, f^{2^{k}}\left(b^{\prime}\right)$ and $f^{2^{k+1}}\left(b^{\prime}\right)$ belong to $f^{j}(\bar{K})$ and they are distinct (same proof as for $a$ ), hence one of them belongs to $f^{j}(K)$, which implies that $b \in f^{j}(K)$. One has $j \neq 0$ because $b \notin J$ and $K \subset J$. But on the other hand $b \in f^{j}\left(L_{k}^{i_{k}}\right) \cap L_{k}^{i_{k}}$, which is empty by Lemma 2.3 , and we get a contradiction. Therefore, $(2)$ is false.

Let $\varepsilon>0$; the negation of (2) implies that there exists $n \geq 0$ such that $\left|I_{n}^{i}\right|<\varepsilon$ for all $0 \leq i<2^{n}$. Let $\delta>0$ be the minimal distance between two distinct intervals among $\left(I_{n}^{i}\right)_{0 \leq i<2^{n}}$. If $x, y \in X$ with $|x-y|<\delta$ then there exists $0 \leq i<2^{n}$ such that $x, y \in I_{n}^{i} \cap \omega\left(x_{0}, f\right)=\omega\left(f^{i}\left(x_{0}\right), f^{2^{n}}\right)$. Thus for all $k \geq 0$ one has $f^{k}(x), f^{k}(y) \in \omega\left(f^{i+k}\left(x_{0}\right), f^{2^{n}}\right) \subset I_{n}^{i+k \text { mod } 2^{n}}$, which implies that $\left|f^{k}(x)-f^{k}(y)\right|<\varepsilon$.

Now we are ready to prove

THEOREM 1.7. Let $f: I \rightarrow I$ be an interval map. If $f$ is Wiggins chaotic then it is Li-Yorke chaotic.

Proof. Suppose that $f$ is not chaotic in the sense of Li-Yorke. By Theorem 1.2 one has $h_{\text {top }}(f)=0$. Consider a closed invariant subset $Y \subset I$ such that $\left.f\right|_{Y}$ is transitive. If $Y$ is finite or has an isolated point, then $\left.f\right|_{Y}$ is not sensitive. If $Y$ is infinite with no isolated point, then there exists $x_{0} \in Y$ such that $\omega\left(x_{0}, f\right)=Y$. By Theorem 2.2, $Y$ does not contain two $f$-non-separable points, and by Lemma $2.6,\left.f\right|_{Y}$ is not sensitive.

3. Wiggins chaos does not imply positive entropy. We are going to build an interval map of zero entropy which is chaotic in the sense of 
Wiggins. It resembles the maps built by Smítal (map $f$ in [23]) and Delahaye (map $g$ in [9]), but we give full details because this construction will be used as a basis for the next example.

Notation. If $I$ is an interval, let $\operatorname{mid}(I)$ denote the middle of $I$. If $f$ is a linear map, let slope $(f)$ denote its constant slope. We write $\uparrow$ (resp. $\downarrow$ ) for "increasing" (resp. "decreasing").

Let $\left(a_{n}\right)_{n \geq 0}$ be an increasing sequence of numbers less than 1 such that $a_{0}=0$. Define $I_{0}^{1}=\left[a_{0}, 1\right]$ and, for all $n \geq 1$,

$$
I_{n}^{0}=\left[a_{2 n-2}, a_{2 n-1}\right], \quad L_{n}=\left[a_{2 n-1}, a_{2 n}\right], \quad I_{n}^{1}=\left[a_{2 n}, 1\right] .
$$

Then $I_{n}^{0} \cup L_{n} \cup I_{n}^{1}=I_{n-1}^{1}$. We fix $\left(a_{n}\right)_{n \geq 0}$ such that the lengths of the intervals $I_{n}^{0}, I_{n}^{1}$ satisfy:

$$
\begin{array}{lll}
\left|I_{n}^{0}\right|=\frac{1}{3^{n}}\left|I_{n-1}^{1}\right|, & \left|I_{n}^{1}\right|=\left(1-\frac{2}{3^{n}}\right)\left|I_{n-1}^{1}\right| & \text { if } n \text { is odd, } \\
\left|I_{n}^{0}\right|=\left(1-\frac{2}{3^{n}}\right)\left|I_{n-1}^{1}\right|, & \left|I_{n}^{1}\right|=\frac{1}{3^{n}}\left|I_{n-1}^{1}\right| & \text { if } n \text { is even, }
\end{array}
$$

This implies that $\left|L_{n}\right|=\left(1 / 3^{n}\right)\left|I_{n-1}^{1}\right|$ for all $n \geq 1$. Note that $\left|I_{n}^{1}\right| \rightarrow 0$, that is, $\lim _{n \rightarrow+\infty} a_{n}=1$; hence $\bigcup_{n \geq 1}\left(I_{n}^{0} \cup L_{n}\right)=[0,1)$.

For all $n \geq 1$, let $\varphi_{n}: I_{n}^{0} \rightarrow I_{n}^{1}$ be the increasing linear homeomorphism mapping $I_{n}^{0}$ onto $I_{n}^{1}$; the slope of $\varphi_{n}$ is slope $\left(\varphi_{n}\right)=\left|I_{n}^{1}\right| /\left|I_{n}^{0}\right|$. Define the map $f:[0,1] \rightarrow[0,1]$ such that $f$ is continuous on $[0,1)$ and

- $f(x)=\varphi_{1}^{-1} \circ \varphi_{2}^{-1} \circ \cdots \circ \varphi_{n-1}^{-1} \circ \varphi_{n}(x)$ for all $x \in I_{n}^{0}, n \geq 1$,

- $\left.f\right|_{L_{n}}$ is linear for all $n \geq 1$,

- $f(1)=0$.

Note that $\left.f\right|_{I_{n}^{0}}$ is linear $\uparrow$. We will show below that $f$ is continuous at 1 .

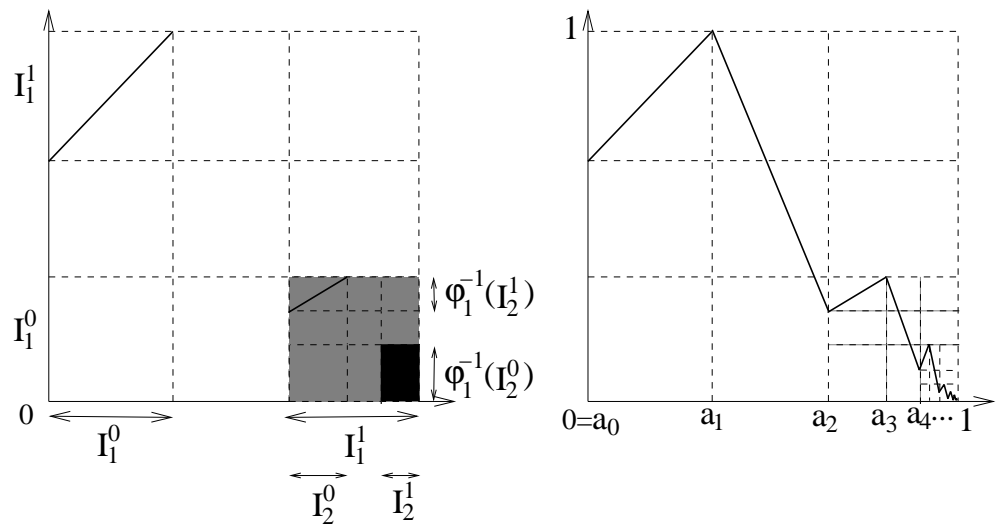

Fig. 1. The first steps of the construction of $f$ (left) and the graph of $f$ (right). This map has a zero entropy and the invariant set $\omega(0, f)$ is transitive and sensitive. 
Let us explain the underlying construction. At step $n=1$ the interval $I_{1}^{0}$ is sent linearly onto $I_{1}^{1}$ (hence $\left.f\right|_{I_{1}^{0}}=\varphi_{1}$ ) and we require that $f\left(I_{1}^{1}\right) \subset I_{1}^{0}$ (grey area in Figure 1). Then we do the same kind of construction in the grey area with respect to $I_{2}^{0}, I_{2}^{1} \subset I_{1}^{1}$ : we rescale $I_{2}^{0}, I_{2}^{1}$ as $\varphi_{1}^{-1}\left(I_{2}^{0}\right), \varphi_{1}^{-1}\left(I_{2}^{1}\right) \subset I_{1}^{0}$ (on the vertical axis) and we send linearly $I_{2}^{0}$ onto $\varphi_{1}^{-1}\left(I_{2}^{1}\right)$; in this way $\left.f\right|_{I_{2}^{0}}=\varphi_{1}^{-1} \circ \varphi_{2}$. We repeat this construction on $I_{2}^{1}$ (black area), and so on. Finally, we fill the gaps in a linear way to get the whole map, which is pictured on the right of Figure 1.

Let $J_{0}^{0}=[0,1]$ and for all $n \geq 1$ define the subintervals $J_{n}^{0}, J_{n}^{1} \subset J_{n-1}^{0}$ by $\min J_{n}^{0}=0, \max J_{n}^{1}=\max J_{n-1}^{0}$ and $\left|J_{n}^{i}\right| /\left|J_{n-1}^{0}\right|=\left|I_{n}^{i}\right| /\left|I_{n-1}^{1}\right|$ for $i=0,1$.

To show that $f$ is continuous at 1 , it is enough to prove that $\max \left(\left.f\right|_{I_{n}^{1}}\right)$ tends to 0 as $n$ goes to infinity. For all $n \geq 1$ one has

$$
\begin{aligned}
\varphi_{n}\left(\max I_{n}^{0}\right) & =\max I_{n}^{1}=1=\min I_{n-1}^{1}+\left|I_{n-1}^{1}\right|, \\
\varphi_{n-1}^{-1} \circ \varphi_{n}\left(\max I_{n}^{0}\right) & =\min I_{n-1}^{0}+\left|I_{n-1}^{1}\right| \operatorname{slope}\left(\varphi_{n-1}^{-1}\right) \\
& =\min I_{n-2}^{1}+\left|I_{n-1}^{1}\right| \operatorname{slope}\left(\varphi_{n-1}^{-1}\right), \\
\varphi_{n-2}^{-1} \circ \varphi_{n-1}^{-1} \circ \varphi_{n}\left(\max I_{n}^{0}\right) & =\min I_{n-2}^{0}+\left|I_{n-1}^{1}\right| \operatorname{slope}\left(\varphi_{n-2}^{-1}\right) \operatorname{slope}\left(\varphi_{n-1}^{-1}\right), \\
\vdots & \\
\varphi_{1}^{-1} \circ \varphi_{2}^{-1} \circ \cdots \circ \varphi_{n-1}^{-1} \circ \varphi_{n}\left(\max I_{n}^{0}\right) & =\min I_{1}^{0}+\left|I_{n-1}^{1}\right| \prod_{i=1}^{n-1} \operatorname{slope}\left(\varphi_{i}^{-1}\right) \\
& =\prod_{i=1}^{n-1} \frac{\left|I_{i}^{0}\right|}{\left|I_{i-1}^{1}\right|}=\left|J_{n-1}^{0}\right| .
\end{aligned}
$$

Consequently,

$$
f\left(\max I_{n}^{0}\right)=\left|J_{n-1}^{0}\right|=\max J_{n-1}^{0} .
$$

According to the definition of $f$, one has $\max \left(\left.f\right|_{I_{n-1}^{1}}\right)=f\left(\max I_{n}^{0}\right)$, so that $\max \left(\left.f\right|_{I_{n-1}^{1}}\right)=\left|J_{n-1}^{0}\right|$. By definition, $\left|J_{n-1}^{0}\right| \leq 1 / 3^{n-2}$, which tends to 0 , and therefore $f$ is continuous at 1 .

The next lemma describes the action of $f$ on the intervals $\left(J_{n}^{i}\right)$ and $\left(I_{n}^{i}\right)$ and collects the properties that we will use later. The interval $I_{n}^{1}$ is periodic of period $2^{n}$ and the map $f^{2^{n-1}}$ swaps $I_{n}^{0}$ and $I_{n}^{1}$. However, we prefer to deal with $J_{n}^{0}=f\left(I_{n}^{1}\right)$; this will simplify the proofs because $\left.f\right|_{I_{n}^{1}}$ is not monotone whereas $\left.f^{i}\right|_{J_{n}^{0}}$ is linear for all $1 \leq i \leq 2^{n}-1$.

Lemma 3.1. Let $f$ be the map defined above. Then for all $n \geq 1$ :

(i) $f\left(I_{n}^{1}\right)=J_{n}^{0}$,

(ii) $f\left(I_{n}^{0}\right)=J_{n}^{1}$, 
(iii) $\left.f^{i}\right|_{J_{n}^{0}}$ is linear $\uparrow$ for all $1 \leq i \leq 2^{n}-1$,

(iv) $f^{2^{n-1}-1}\left(J_{n}^{0}\right)=I_{n}^{0}$ and $f^{2^{n}-1}\left(J_{n}^{0}\right)=I_{n}^{1}$,

(v) $f^{i}\left(J_{n}^{0}\right) \subset \bigcup_{1 \leq k \leq n} I_{k}^{0}$ for all $0 \leq i \leq 2^{n}-2$,

(vi) $\left(f^{i}\left(J_{n}^{0}\right)\right)_{0 \leq i<2^{n}}$ are pairwise disjoint;

and the previous clauses also imply

(vii) $f^{2^{n-1}}\left(J_{n}^{0}\right)=J_{n}^{1}$,

(viii) $f^{2^{n}}\left(J_{n}^{0}\right)=J_{n}^{0}$,

(ix) $\left.f^{2^{n-1}}\right|_{I_{n}^{0}}$ is linear $\uparrow$ and $f^{2^{n-1}}\left(I_{n}^{0}\right)=I_{n}^{1}$,

(x) $f^{2^{n-1}}\left(I_{n}^{1}\right)=I_{n}^{0}$

(xi) $\left(f^{i}\left(I_{n}^{0}\right)\right)_{0 \leq i<2^{n}}$ are pairwise disjoint and $f^{2^{n}}\left(I_{n}^{1}\right)=I_{n}^{1}$.

Proof. According to $(3), \max \left(\left.f\right|_{I_{n}^{1}}\right)=f\left(\max I_{n+1}^{0}\right)=\max J_{n}^{0}$; moreover $f(1)=0=\min J_{n}^{0}$. Thus $f\left(I_{n}^{1}\right)=J_{n}^{0}$ by continuity; this is (i).

According to the definition of $f$,

$$
\begin{aligned}
\left|f\left(I_{n}^{0}\right)\right| & =\left|I_{n}^{0}\right| \operatorname{slope}\left(\varphi_{n}\right) \prod_{i=1}^{n-1} \operatorname{slope}\left(\varphi_{i}^{-1}\right) \\
& =\left|I_{n}^{1}\right| \prod_{i=1}^{n-1} \frac{\left|I_{i}^{0}\right|}{\left|I_{i}^{1}\right|}=\frac{\left|I_{n}^{1}\right|}{\left|I_{n-1}^{1}\right|} \prod_{i=1}^{n-1} \frac{\left|I_{i}^{0}\right|}{\left|I_{i-1}^{1}\right|}=\left|J_{n}^{1}\right| .
\end{aligned}
$$

Moreover $f\left(\max I_{n}^{0}\right)=\max J_{n-1}^{0}=\max J_{n}^{1}$ by $(3)$, and thus $f\left(I_{n}^{0}\right)=J_{n}^{1}$. This gives (ii).

We show by induction on $n$ that (iii) and (iv) are satisfied.

- This is true for $n=1$ because $J_{1}^{0}=I_{1}^{0}, J_{1}^{1}=I_{1}^{1},\left.f\right|_{I_{1}^{0}}=\varphi_{1}$ is linear $\uparrow$ and $f\left(I_{1}^{0}\right)=I_{1}^{1}$.

- Suppose that (iii) and (iv) are true for $n$. Since $J_{n+1}^{0} \subset J_{n}^{0}$, the map $\left.f^{i}\right|_{J_{n+1}^{0}}$ is linear $\uparrow$ for all $1 \leq i \leq 2^{n}-1$ and $f^{2^{n}-1}\left(J_{n+1}^{0}\right) \subset I_{n}^{1}$; moreover the linearity implies that

$$
\min f^{2^{n}-1}\left(J_{n+1}^{0}\right)=\min f^{2^{n}-1}\left(J_{n}^{0}\right)=\min I_{n}^{1}=\min I_{n+1}^{0}
$$

and

$$
\frac{\left|f^{2^{n}-1}\left(J_{n+1}^{0}\right)\right|}{\left|I_{n}^{1}\right|}=\frac{\left|J_{n+1}^{0}\right|}{\left|J_{n}^{0}\right|}=\frac{\left|I_{n+1}^{0}\right|}{\left|I_{n}^{1}\right|} .
$$

Therefore $f^{2^{n}-1}\left(J_{n+1}^{0}\right)=I_{n+1}^{0}$. Then $f^{2^{n}}\left(J_{n+1}^{0}\right)=J_{n+1}^{1}$ by (ii). Since $J_{n+1}^{1} \subset J_{n}^{0}$, the induction hypothesis applies: $\left.f^{i}\right|_{J_{n+1}^{1}}$ is linear $\uparrow$ for all $1 \leq i \leq 2^{n}-1, f^{2^{n}-1}\left(J_{n+1}^{1}\right) \subset I_{n}^{1}$, and by linearity

$$
\max f^{2^{n}-1}\left(J_{n+1}^{1}\right)=\max f^{2^{n}-1}\left(J_{n}^{0}\right)=1=\max I_{n+1}^{1}
$$

and $\left|f^{2^{n}-1}\left(J_{n+1}^{1}\right)\right|=\left|I_{n+1}^{1}\right|$, hence $f^{2^{n+1}-1}\left(J_{n+1}^{0}\right)=f^{2^{n}-1}\left(J_{n+1}^{1}\right)=$ $I_{n+1}^{1}$. This gives (iii) and (iv) for $n+1$. 
Now we prove (v) by induction on $n$ :

- This is true for $n=1$ because $J_{1}^{0}=I_{1}^{0}$.

- Suppose that (v) is true for $n$. Since $J_{n+1}^{0} \subset J_{n}^{0}$ it follows that $f^{i}\left(J_{n+1}^{0}\right)$ $\subset \bigcup_{1 \leq k \leq n} I_{k}^{n}$ for all $0 \leq i<2^{n}-1$. Moreover $f^{2^{n}-1}\left(J_{n+1}^{0}\right)=I_{n+1}^{0}$ by (iv), and $f^{2^{n}}\left(J_{n+1}^{0}\right)=f\left(I_{n+1}^{0}\right)=J_{n+1}^{1}$ by (ii). Since $J_{n+1}^{1} \subset J_{n}^{0}$ we can use the induction hypothesis again to get $f^{2^{n}+i}\left(J_{n+1}^{0}\right) \subset \bigcup_{1 \leq k \leq n} I_{k}^{n}$ for all $0 \leq i<2^{n}-1$. This gives (v) for $n+1$.

Next we prove (vi). Suppose that $f^{i}\left(J_{n}^{0}\right) \cap f^{j}\left(J_{n}^{0}\right) \neq \emptyset$ for some $0 \leq$ $i<j<2^{n}$. Then $f^{2^{n}-1-j}\left(f^{i}\left(J_{n}^{0}\right)\right) \cap f^{2^{n}-1-j}\left(f^{j}\left(J_{n}^{0}\right)\right) \neq \emptyset$. But $f^{2^{n}-1}\left(J_{n}^{0}\right)=$ $I_{n}^{1}$ by (iv) and $f^{2^{n}-1-(j-i)}\left(J_{n}^{0}\right) \subset\left[0, \max I_{n}^{0}\right]$ by $(\mathrm{v})$, so these two sets are disjoint, which is a contradiction.

Finally, we indicate how to obtain the other assertions. Assertions (vii) and (viii) follow respectively from (iv) +(ii) and (iv)+(i). Assertion (ix) follows from (iii)+(iv). Assertion (x) follows from (i)+(iv). Assertion (xi) follows from the combination of (i), (iv) and (vi).

Define $K_{n}=\bigcup_{i \geq 0} f^{i}\left(I_{n}^{1}\right)$ for all $n \geq 0$ and $K=\bigcap_{n \geq 0} K_{n}$. According to Lemma 3.1, $K_{n}$ is the disjoint union of the intervals $\left(f^{i}\left(J_{n}^{0}\right)\right)_{0 \leq i \leq 2^{n}-1}$. The set $K$ has a Cantor-like construction: at each step a middle part of every connected component of $K_{n}$ is removed to get $K_{n+1}$. However, $K$ is not a Cantor set because its interior is not empty (see Proposition 3.3). Proposition 3.2 states that the entropy of $f$ is null. Next we show in Proposition 3.3 that the set $\omega(0, f)$ contains $\partial K$. Then we prove that $\omega(0, f)$ is transitive and sensitive to initial conditions.

Proposition 3.2. Let $f$ be the map defined above. Then $h_{\mathrm{top}}(f)=0$.

Proof. By definition the restriction $\left.f\right|_{L_{n}}$ is linear decreasing and thus $f\left(L_{n}\right) \subset\left[0, f\left(\max I_{n}^{0}\right)\right]$. Moreover $f\left(\max I_{n}^{0}\right)=\max J_{n-1}^{0}$ by $(3)$, so that $f\left(L_{n}\right) \subset J_{n-1}^{0}$. Then Lemma 3.1(iii) implies that $\left.f^{2^{n-1}}\right|_{L_{n}}$ is linear decreasing. Since $\left.f^{2^{n-1}}\right|_{I_{n}^{0}}$ is linear increasing and $f^{2^{n-1}}\left(I_{n}^{0}\right)=I_{n}^{1}$ by Lemma 3.1(ix), it follows that $f^{2^{n-1}}\left(\min L_{n}\right)=\max I_{n}^{1}=1$; moreover $f^{2^{n-1}}\left(I_{n}^{1}\right)=I_{n}^{0}$ by Lemma $3.1(\mathrm{x})$, and thus $f^{2^{n-1}}\left(\max L_{n}\right) \in I_{n}^{0}$. We deduce that $f^{2^{n-1}}\left(L_{n}\right) \supset$ $L_{n} \cup I_{n}^{1}$, and by Lemma 2.4 there exists $z_{n} \in L_{n}$ such that $f^{2^{n-1}}\left(z_{n}\right)=z_{n}$. The period of $z_{n}$ is exactly $2^{n-1}$ because $L_{n} \subset I_{n-1}^{1}$ and the intervals $\left(f^{i}\left(I_{n-1}^{1}\right)\right)_{0 \leq i<2^{n}}$ are pairwise disjoint by Lemma $3.1(\mathrm{xi})$.

By definition $\left|L_{n}\right| \leq\left|I_{n}^{1}\right|$, hence slope $\left(\left.f^{2^{n-1}}\right|_{L_{n}}\right) \leq-2$. If the points $x, f^{2^{n-1}}(x), \ldots, f^{k 2^{n-1}}(x)$ belong to $L_{n}$ then $\left|f^{(k+1) 2^{n-1}}(x)-z_{n}\right| \geq 2^{k}\left|x-z_{n}\right|$; thus, for all $x \in L_{n}, x \neq z_{n}$, there exists $k \geq 1$ such that $f^{k 2^{n-1}}(x) \notin L_{n}$. Since $I_{n-1}^{1}=I_{n}^{0} \cup L_{n} \cup I_{n}^{1}$ and $f^{2^{n-1}}\left(I_{n-1}^{1}\right)=I_{n-1}^{1}$ by Lemma 3.1(xi), this implies that $f^{k 2^{n-1}}(x) \in I_{n}^{0} \cup I_{n}^{1}$. In addition $f^{2^{n-1}}\left(I_{n}^{0}\right)=I_{n}^{1}$ by Lemma 3.1(ix), 
and therefore

$$
\forall x \in I_{n-1}^{1}, x \neq z_{n}, \exists k \geq 0, \quad f^{k}(x) \in I_{n}^{1} .
$$

Starting with $I_{0}^{1}=[0,1]$, a straightforward induction shows that, for all $x \in[0,1]$, if the orbit of $x$ does not meet $\left\{z_{n} \mid n \geq 1\right\}$ then for all integers $n \geq 1$ there exists $k \geq 0$ such that $f^{k}(x) \in I_{n}^{1}$; in particular $\omega(x, f) \subset K$. According to Lemma 3.1(xi) the set $K$ contains no periodic point because $K \subset \bigcup_{i \geq 0} f^{i}\left(I_{n}^{1}\right)$ for all $n \geq 1$; thus every periodic point is in the orbit of some $z_{n}$, and therefore its period is a power of 2 . Finally, $h_{\text {top }}(f)=0$ by Theorem 1.9.

The orbit of 0 obviously enters $f^{i}\left(J_{n}^{0}\right)$ for all $n \geq 0$ and $0 \leq i<2^{n}$, and so $\omega(0, f)$ meets all connected components of $K$. We show in the next lemma that $\omega(0, f)$ contains $\partial K$; the proof relies on the idea that the smaller of the intervals $J_{n+1}^{0}$ and $J_{n+1}^{1}$ contains alternately either $\min J_{n}^{0}$ or $\max J_{n}^{0}$ when $n$ varies, so that both endpoints of a connected component of $K$ can be approximated by small intervals of the form $f^{i}\left(J_{n}^{0}\right)$.

Proposition 3.3. Let $f$ and $K$ be as defined above. Then $\partial K \subset \omega(0, f)$. In particular $\omega(0, f)$ is infinite and contains 0 , and $\left.f\right|_{\omega(0, f)}$ is transitive.

Proof. According to the definition of $K$, the connected components of $K$ are exactly the non-empty sets of the form $\bigcap_{n \geq 0} f^{j_{n}}\left(J_{n}^{0}\right)$ with $0 \leq j_{n}<2^{n}$. Let $y$ be a point in $\partial K$. For all $n \geq 0$ there exists $0 \leq j_{n}<2^{n}$ such that $y \in f^{j_{n}}\left(J_{n}^{0}\right)$, and there exists a sequence $\left(y_{n}\right)_{n \geq 0}$ such that $y=\lim _{n \rightarrow+\infty} y_{n}$ and $y_{n} \in \partial f^{j_{n}}\left(J_{n}^{0}\right)=\left\{\min f^{j_{n}}\left(J_{n}^{0}\right), \max f^{j_{n}}\left(J_{n}^{0}\right)\right\}$. Let $\varepsilon>0$ and $N \geq 0$. Let $n$ be an even integer such that $1 / 3^{n+1}<\varepsilon$ and $\left|y_{n}-y\right|<\varepsilon$, and let $k \geq 0$ be such that $k 2^{n+1} \geq N$.

First, suppose that $y_{n}=\min f^{j_{n}}\left(J_{n}^{0}\right)$. Since $0 \in J_{n+1}^{0}$ and $f^{2^{n+1}}\left(J_{n+1}^{0}\right)=$ $J_{n+1}^{0}$ by Lemma 3.1(viii), it follows that $f^{k 2^{n+1}+j_{n}}(0) \in f^{j_{n}}\left(J_{n+1}^{0}\right)$. By Lemma 3.1(iii) one has $\min f^{j_{n}}\left(J_{n+1}^{0}\right)=\min f^{j_{n}}\left(J_{n}^{0}\right)=y_{n}$ and

$$
\frac{\left|f^{j_{n}}\left(J_{n+1}^{0}\right)\right|}{\left|f^{j_{n}}\left(J_{n}^{0}\right)\right|}=\frac{\left|J_{n+1}^{0}\right|}{\left|J_{n}^{0}\right|}=\frac{1}{3^{n+1}}<\varepsilon,
$$

and therefore $\left|f^{k 2^{n+1}+j_{n}}(0)-y_{n}\right|<\varepsilon\left|f^{j_{n}}\left(J_{n}^{0}\right)\right| \leq \varepsilon$.

Secondly, suppose that $y_{n}=\max f^{j_{n}}\left(J_{n}^{0}\right)$. The point $f^{k 2^{n+2}}(0)$ belongs to $J_{n+2}^{0}$ and $f^{2^{n+1}}\left(J_{n+2}^{0}\right)=J_{n+2}^{1}$ by Lemma 3.1 (vii), so

$$
f^{k 2^{n+2}+2^{n+1}+2^{n}+j_{n}}(0) \in f^{2^{n}+j_{n}}\left(J_{n+2}^{1}\right) .
$$

According to Lemma 3.1(iii)-(vii) one has $\max f^{2^{n}+j_{n}}\left(J_{n+2}^{1}\right)=\max f^{2^{n}+j_{n}}\left(J_{n+1}^{0}\right)=\max f^{j_{n}}\left(J_{n+1}^{1}\right)=\max f^{j_{n}}\left(J_{n}^{0}\right)=y_{n}$. Moreover

$$
f^{2^{n}}\left(J_{n+2}^{1}\right) \subset f^{2^{n}}\left(J_{n+1}^{0}\right)=J_{n+1}^{1} \subset J_{n}^{0} .
$$


Thus

$$
\frac{\left|f^{j_{n}+2^{n}}\left(J_{n+2}^{1}\right)\right|}{\left|f^{j_{n}}\left(J_{n}^{0}\right)\right|}=\frac{\left|f^{2^{n}}\left(J_{n+2}^{1}\right)\right|}{\left|J_{n}^{0}\right|}=\frac{\left|f^{2^{n}}\left(J_{n+2}^{1}\right)\right|}{\left|f^{2^{n}}\left(J_{n+1}^{0}\right)\right|} \cdot \frac{\left|J_{n+1}^{1}\right|}{\left|J_{n}^{0}\right|}=\frac{1}{3^{n+2}}\left(1-\frac{2}{3^{n+1}}\right) \text {. }
$$

Consequently, $\left|f^{k 2^{n+2}+2^{n+1}+2^{n}+j_{n}}(0)-y_{n}\right| \leq\left|f^{j_{n}+2^{n}}\left(J_{n+2}^{1}\right)\right|<\varepsilon$.

In both cases there exists $p \geq N$ such that $\left|f^{p}(0)-y_{n}\right|<\varepsilon$, hence $\left|f^{p}(0)-y\right|<2 \varepsilon$. This means that $y \in \omega(0, f)$, that is, $\partial K \subset \omega(0, f)$. The point $\{0\}=\bigcap_{n \geq 0} J_{n}^{0}$ belongs to $\partial K$, so $0 \in \omega(0, f)$ and $\left.f\right|_{\omega(0, f)}$ is transitive. Finally, $K_{n}$ has $2^{n}$ connected components, each of which contains two connected components of $K_{n+1}$; thus $K$ has an infinite number of connected components, which implies that $\partial K$ is infinite.

In the proof of the next proposition, we first show that $K$ contains a non-degenerate connected component $B$.

Proposition 3.4. Let $f$ be the map defined above. Then $\left.f\right|_{\omega(0, f)}$ is sensitive to initial conditions.

Proof. First we define by induction a sequence of intervals $B_{n}=f^{i_{n}}\left(J_{n}^{0}\right)$ for some $0 \leq i_{n}<2^{n}$ such that $B_{n} \subset B_{n-1}$ and $\left|B_{n}\right|=\left(1-2 / 3^{n}\right)\left|B_{n-1}\right|$ for all $n \geq 1$.

- Take $B_{0}=J_{0}=[0,1]$.

- Suppose that $B_{n-1}=f^{i_{n-1}}\left(J_{n-1}^{0}\right)$ is already built. If $n$ is even, take $i_{n}=i_{n-1}$ and $B_{n}=f^{i_{n}}\left(J_{n}^{0}\right)$. The map $\left.f^{i_{n-1}}\right|_{J_{n-1}^{0}}$ is linear $\uparrow$ by Lemma 3.1(iii) and $J_{n}^{0} \subset J_{n-1}^{0}$, so that

$$
\frac{\left|B_{n}\right|}{\left|B_{n-1}\right|}=\frac{\left|J_{n}^{0}\right|}{\left|J_{n-1}^{0}\right|}=1-\frac{2}{3^{n}} \text {. }
$$

If $n$ is odd, take $i_{n}=i_{n-1}+2^{n-1}$ and $B_{n}=f^{i_{n}}\left(J_{n}^{0}\right)$. According to Lemma 3.1(vii)-(iii) one has $B_{n}=f^{i_{n-1}}\left(J_{n}^{1}\right)$ and $\left.f^{i_{n-1}}\right|_{J_{n-1}^{0}}$ is linear $\uparrow$, so

$$
\frac{\left|B_{n}\right|}{\left|B_{n-1}\right|}=\frac{\left|J_{n}^{1}\right|}{\left|J_{n-1}^{0}\right|}=1-\frac{2}{3^{n}}
$$
because

Let $B=\bigcap_{n \geq 0} B_{n}$. This is a compact interval and it is non-degenerate

$$
\log |B|=\log \left|B_{0}\right|+\sum_{n \geq 1} \log \left(1-\frac{2}{3^{n}}\right)>-\infty .
$$

Moreover $B$ is a connected component of $K$, so $\partial B \subset \partial K$. Let $b_{0}=\min B$ and $b_{1}=\max B$; one has $b_{0}, b_{1} \in \omega(0, f)$ by Proposition 3.3 .

The set $\omega(0, f)$ is included in the periodic orbit of $J_{n}^{0}$, and consequently $f^{k}\left(J_{n}^{0} \cap \omega(0, f)\right)=f^{k}\left(J_{n}^{0}\right) \cap \omega(0, f)$ for all $k \geq 0$. Let $\varepsilon>0$ and $k \geq 1$. There exists $n \geq 0$ such that $\left|J_{n}^{0}\right|<\varepsilon$ and there exist $x_{0}, x_{1} \in J_{n}^{0} \cap \omega(0, f)$ such that 
$f^{i_{n}+k 2^{n}}\left(x_{0}\right)=b_{0}$ and $f^{i_{n}+k 2^{n}}\left(x_{1}\right)=b_{1}$. Let $\delta=\left|b_{1}-b_{0}\right| / 4$. The triangular inequality implies that either $\left.\mid f^{i_{n}+k 2^{n}}(0)-b_{0}\right) \mid \geq 2 \delta$ or $\left|f^{i_{n}+k 2^{n}}(0)-b_{1}\right| \geq 2 \delta$. In other words, for all $\varepsilon>0$ and $k \geq 1$ there exist $x \in[0, \varepsilon] \cap \omega(0, f)$ and $i \geq k$ such that $\left|f^{i}(0)-f^{i}(x)\right| \geq 2 \delta$. Let $y \in \omega(0, f)$ and $\varepsilon>0$; there exists $k \geq 0$ such that $\left|f^{k}(0)-y\right|<\varepsilon / 2$. By continuity of $f^{k}$ there is $\eta>0$ such that $f^{k}([0, \eta]) \subset[y-\varepsilon, y+\varepsilon]$. What precedes shows that there exist $x \in[0, \eta] \cap \omega(0, f)$ and $i>k$ such that $\left|f^{i}(0)-f^{i}(x)\right| \geq 2 \delta$, and if $z=f^{k}(x), z^{\prime}=f^{k}(0)$ and $j=i-n$ we find that $z, z^{\prime} \in[y-\varepsilon, y+\varepsilon]$ and $\left|f^{j}(z)-f^{j}\left(z^{\prime}\right)\right| \geq 2 \delta$. Then the triangular inequality implies that either $\left|f^{j}(y)-f^{j}(z)\right| \geq \delta$ or $\left|f^{j}(y)-f^{j}\left({ }^{\prime} z\right)\right| \geq \delta$. We conclude that $\left.f\right|_{\omega(0, f)}$ is sensitive to initial conditions.

Finally, Propositions 3.2, 3.3 and 3.4 give Theorem 1.6.

Remark 3.5. According to Theorem 1.7 and Proposition 3.4, the map $f$ is chaotic in the sense of Li-Yorke. It can be proven directly that $b_{0}, b_{1}$ are $f$-non-separable, so Theorem 2.2 applies.

4. Li-Yorke chaos does not imply Wiggins chaos. The aim of this section is to exhibit an interval map which is chaotic in the sense of $\mathrm{Li}-$ Yorke but has no transitive sensitive subsystem. This map resembles the one of Section 3: the construction on the set $\bigcup I_{n}^{0}$ is the same except that the lengths of the intervals differ; the dynamics on $L_{n}$ is different.

4.1. Definition of the map $g$. We are going to build a continuous map $g:[0,3 / 2] \rightarrow[0,3 / 2]$. Let $\left(a_{n}\right)_{n \geq 0}$ be an increasing sequence of numbers less than 1 such that $a_{0}=0$. Define $I_{0}^{1}=\left[a_{0}, 1\right]$ and for all $n \geq 1$,

$$
I_{n}^{0}=\left[a_{2 n-2}, a_{2 n-1}\right], \quad L_{n}=\left[a_{2 n-1}, a_{2 n}\right], \quad I_{n}^{1}=\left[a_{2 n}, 1\right] .
$$

Then $I_{n}^{0} \cup L_{n} \cup I_{n}^{1}=I_{n-1}^{1}$.

Fix $\left(a_{n}\right)_{n \geq 0}$ such that the lengths of the intervals satisfy

$$
\forall n \geq 1, \quad\left|I_{n}^{0}\right|=\left|L_{n}\right|=\frac{1}{3^{n}}\left|I_{n-1}^{1}\right| \quad \text { and } \quad\left|I_{n}^{1}\right|=\left(1-\frac{2}{3^{n}}\right)\left|I_{n-1}^{1}\right| .
$$

Let $a=\lim _{n \rightarrow+\infty} a_{n}$. Then $\bigcup_{n \geq 1}\left(I_{n}^{0} \cup L_{n}\right)=[0, a)$ and $a<1$ because

$$
\log (1-a)=\sum_{n=1}^{+\infty} \log \left(1-\frac{2}{3^{n}}\right)>-\infty .
$$

For all $n \geq 1$, let $\varphi_{n}: I_{n}^{0} \rightarrow I_{n}^{1}$ be the increasing linear homeomorphism mapping $I_{n}^{0}$ onto $I_{n}^{1}$. Define the map $g:[0,3 / 2] \rightarrow[0,3 / 2]$ such that $g$ is continuous on $[0,3 / 2] \backslash\{a\}$ and

- $g(x)=\varphi_{1}^{-1} \circ \varphi_{2}^{-2} \circ \cdots \circ \varphi_{n-1}^{-1} \circ \varphi_{n}(x)$ for all $x \in I_{n}^{0}, n \geq 1$,

- $g$ is linear $\uparrow$ of slope $\lambda_{n}$ on $\left[\min L_{n}, \operatorname{mid}\left(L_{n}\right)\right]$ for all $n \geq 1$, 
- $g$ is linear $\downarrow$ on $\left[\operatorname{mid}\left(L_{n}\right), \max L_{n}\right]$ for all $n \geq 1$,

- $g(x)=0$ for all $x \in[a, 1]$,

- $g(x)=x-1$ for all $x \in[1,3 / 2]$,

where the slopes $\left(\lambda_{n}\right)$ will be defined below. We will also show that $g$ is continuous at $a$. The map $g$ is pictured in Figure 2 .

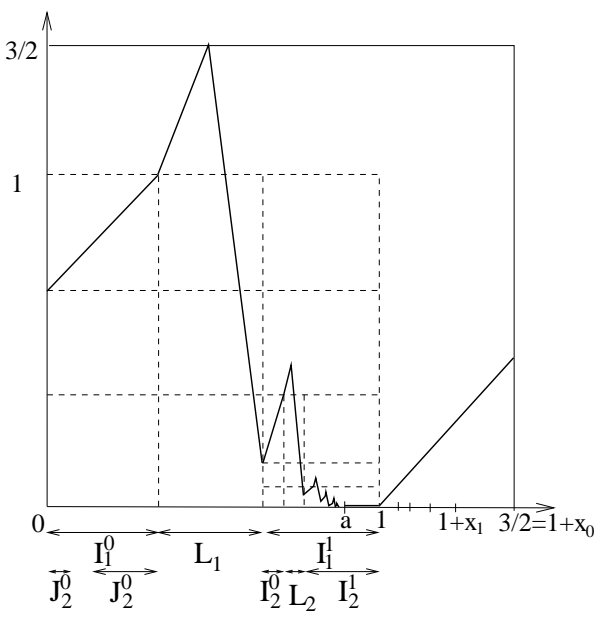

Fig. 2. The graph of $g$; this map is Li-Yorke chaotic but not Wiggins chaotic.

Let $J_{0}^{0}=[0,1]$ and for all $n \geq 1$ define the subintervals $J_{n}^{0}, J_{n}^{1} \subset J_{n-1}^{0}$ such that $\min J_{n}^{0}=0, \max J_{n}^{1}=\max J_{n-1}^{0}$ and $\left|J_{n}^{i}\right| /\left|J_{n-1}^{0}\right|=\left|I_{n}^{i}\right| /\left|I_{n-1}^{1}\right|$ for $i=0,1$; let $M_{n}=\left[\max J_{n}^{0}, \min J_{n}^{1}\right]$.

Note that on $\bigcup_{n \geq 1} I_{n}^{0}$ the map $g$ is defined similarly to the map $f$ of Section 3, and therefore the assertions of Lemma 3.1 remain valid for $g$, except (i) and its derived results (viii), (x), (xi).

Lemma 4.1. Let $g$ be the map defined above. Then for all $n \geq 1$ :

(i) $g\left(I_{n}^{0}\right)=J_{n}^{1}$,

(ii) $\left.g^{i}\right|_{J_{n}^{0}}$ is linear $\uparrow$ for all $0 \leq i \leq 2^{n}-1$,

(iii) $g^{2^{n-1}-1}\left(J_{n}^{0}\right)=I_{n}^{0}$ and $g^{2^{n}-1}\left(J_{n}^{0}\right)=I_{n}^{1}$,

(iv) $g^{i}\left(J_{n}^{0}\right) \subset \bigcup_{1 \leq k \leq n} I_{k}^{0}$ for all $0 \leq i \leq 2^{n}-2$,

(v) $\left(g^{i}\left(J_{n}^{0}\right)\right)_{0 \leq i<2^{n}}$ are pairwise disjoint.

(vi) $\left.g^{2^{n-1}}\right|_{I_{n}^{0}}$ is linear $\uparrow$ and $g^{2^{n-1}}\left(I_{n}^{0}\right)=I_{n}^{1}$,

(vii) $\left.g^{2^{n-1}-1}\right|_{M_{n}}$ is linear $\uparrow$ and $g^{2^{n-1}-1}\left(M_{n}\right)=L_{n}$,

(viii) $g\left(\min L_{n}\right)=\min M_{n-1}$,

(ix) $g^{2^{n-2}}\left(\min L_{n}\right)=\min L_{n-1}$.

Proof. For assertions (i) to (vi) see the proof of Lemma 3.1. 
According to (ii), the map $\left.g^{2^{n-1}-1}\right|_{M_{n}}$ is linear $\uparrow$ because $M_{n}$ is included in $J_{n-1}^{0}$. Since $M_{n}=\left[\max J_{n}^{0}\right.$, $\left.\min J_{n}^{1}\right]$ and $L_{n}=\left[\max I_{n}^{0}\right.$, $\left.\min I_{n}^{1}\right]$, assertions (i), (ii) and (iii) imply that $g^{2^{n-1}-1}\left(M_{n}\right)=L_{n}$, which is (vii).

The map $\left.g\right|_{I_{n}^{0}}$ is increasing and $\min L_{n}=\max I_{n}^{0}$, hence, according to (i), one has $g\left(\min L_{n}\right)=\max J_{n}^{1}=\max J_{n-1}^{0}=\min M_{n-1}$; this is (viii).

Finally, (vii) and (viii) imply (ix).

For all $n \geq 0$, define $x_{n}=\operatorname{mid}\left(M_{n+1}\right)$, that is, $x_{n}=\frac{3}{2} \prod_{i=1}^{n+1} 1 / 3^{i}$. It is a decreasing sequence and $x_{0}=1 / 2$; therefore, for all $n \geq 0, g\left(1+x_{n}\right)$ is well defined and equal to $x_{n}$.

For all $n \geq 0$ let $t_{n}=\operatorname{slope}\left(\left.g^{2^{n}-1}\right|_{J_{n}^{0}}\right)$; by convention $g^{0}$ is the identity map so $t_{0}=1$. Fix $\lambda_{1}=2 x_{1} /\left|L_{1}\right|$ and for all $n \geq 2$ define inductively $\lambda_{n}$ such that

$$
\frac{\left|L_{n}\right|}{2} \prod_{i=1}^{n} \lambda_{i} \prod_{i=0}^{n-2} t_{i}=x_{n} .
$$

By convention an empty product is equal to 1 , so (4) is satisfied for $n=1$.

The slopes $\left(\lambda_{n}\right)_{n \geq 1}$ satisfy $g^{2^{n-1}}\left(\left[\min L_{n}, \operatorname{mid}\left(L_{n}\right)\right]\right)=\left[1,1+x_{n}\right]$, as proven in the next lemma. This means that under the action of $g^{2^{n-1}}$ the image of $L_{n}$ falls outside of $[0,1]$ but remains close to 1 . We also list properties of $g$ on the intervals $L_{n}, I_{n}^{1}$ and $\left[1,1+x_{n}\right]$.

Lemma 4.2. Let $g$ be the map defined above. Then:

(i) $\left.g^{2^{n}}\right|_{\left[1,1+x_{n}\right]}$ is linear $\uparrow$ and $g^{2^{n}}\left(\left[1,1+x_{n}\right]\right)=\left[\min I_{n+1}^{0}, \operatorname{mid}\left(L_{n+1}\right)\right]$ for all $n \geq 0$

(ii) $\left.g^{2^{n-1}}\right|_{\left[\min L_{n}, \operatorname{mid}\left(L_{n}\right)\right]}$ is linear $\uparrow$ and $g^{2^{n-1}}\left(\left[\min L_{n}, \operatorname{mid}\left(L_{n}\right)\right]\right)=$ $\left[1,1+x_{n}\right]$ for all $n \geq 1$,

(iii) $\left.g^{2^{n+1}}\right|_{\left[1,1+x_{n}\right]}$ is $\uparrow$ and $g^{2^{n+1}}\left(\left[1,1+x_{n}\right]\right)=I_{n+1}^{1} \cup\left[1,1+x_{n+1}\right]$ for all $n \geq 1$,

(iv) $g\left(I_{n}^{1}\right) \subset\left[0, \operatorname{mid}\left(M_{n}\right)\right]$ for all $n \geq 1$,

(v) $g^{2^{n}}\left(\left[\min I_{n}^{1}, 1+x_{n}\right]\right) \subset\left[\min I_{n}^{1}, 1+x_{n}\right]$ and $g^{i}\left(\left[\min I_{n}^{1}, 1+x_{n}\right]\right) \subset[0,1]$ for all $1 \leq i \leq 2^{n}-1, n \geq 1$.

Proof. The map $\left.g\right|_{\left[1,1+x_{n}\right]}$ is linear $\uparrow$ and $g\left(\left[1,1+x_{n}\right]\right)=\left[0, \operatorname{mid}\left(M_{n+1}\right)\right]$ $\subset J_{n}^{0}$, so $\left.g^{2^{n}}\right|_{\left[1,1+x_{n}\right]}$ is linear $\uparrow$ by Lemma 4.1(ii). Moreover $g^{2^{n}-1}(0)=$ $\min I_{n+1}^{0}$ and $g^{2^{n}-1}\left(\operatorname{mid}\left(M_{n+1}\right)\right)=\operatorname{mid}\left(L_{n+1}\right)$ by Lemma $4.1($ iii $)+($ iv $)$, which gives (i).

Before proving (ii) we show some intermediate results. Let $n \geq 2$ and $2 \leq k \leq n$. Then

$$
\begin{aligned}
\lambda_{n} \cdots \lambda_{k} \cdot t_{n-2} \cdots t_{k-2} & =\frac{\prod_{i=1}^{n} \lambda_{i} \prod_{i=0}^{n-2} t_{i}}{\prod_{i=1}^{k-1} \lambda_{i} \prod_{i=0}^{k-3} t_{i}} \\
& =\frac{x_{n} /\left|L_{n}\right|}{x_{k-1} /\left|L_{k-1}\right|}
\end{aligned}
$$




$$
\begin{aligned}
& =\prod_{i=k+1}^{n+1} \frac{1}{3^{i}} \prod_{i=k-1}^{n-1} \frac{1}{1-2 / 3^{i}} \cdot \frac{3^{n}}{3^{k-1}} \\
& =\frac{1}{3^{n-k+1}} \prod_{i=k-1}^{n-1} \frac{1}{3^{i}-2}
\end{aligned}
$$

and so

$$
\lambda_{n} \cdots \lambda_{k} \cdot t_{n-2} \cdots t_{k-2}<1 .
$$

By definition, $g\left(\operatorname{mid}\left(L_{n}\right)\right)=g\left(\min L_{n}\right)+\lambda_{n}\left|L_{n}\right| / 2$, and by $(4)$,

$$
\begin{aligned}
\lambda_{n} \frac{\left|L_{n}\right|}{2} & =\frac{x_{n}}{t_{n-2} \prod_{i=1}^{n-1} \lambda_{i} \prod_{i=0}^{n-3} t_{i}}=\frac{x_{n}\left|L_{n-1}\right|}{2 x_{n-1} t_{n-2}} \\
& =\frac{1}{3^{n+1}} \frac{\left|M_{n-1}\right|}{2} \quad \text { because } t_{n-2}=\frac{\left|L_{n-1}\right|}{\left|M_{n-1}\right|} \text { by Lemma 4.1(vii) } \\
& <\frac{\left|M_{n-1}\right|}{2} .
\end{aligned}
$$

Moreover $g\left(\min L_{n}\right)=\min M_{n-1}$ by Lemma 4.1 (viii), hence

(6) $g\left(\left[\min L_{n}, \operatorname{mid}\left(L_{n}\right)\right]\right) \subset\left[\min M_{n-1}, \operatorname{mid}\left(M_{n-1}\right)\right] \quad$ for all $n \geq 2$.

We show by induction on $k=n, \ldots, 2$ that

- the map $g^{2^{n-2}+2^{n-3}+\cdots+2^{k-2}}$ is linear $\uparrow$ of slope $\lambda_{n} \cdots \lambda_{k} t_{n-2} \cdots t_{k-2}$ on $\left[\min L_{n}, \operatorname{mid}\left(L_{n}\right)\right]$ and $\operatorname{maps} \min L_{n}$ to $\min L_{k-1}$,

$-g^{i}\left(\left[\min L_{n}, \operatorname{mid}\left(L_{n}\right)\right]\right) \subset[0,1]$ for all $0 \leq i \leq 2^{n-2}+2^{n-3}+\cdots+2^{k-2}$.

- By (6) one has

$$
g\left(\left[\min L_{n}, \operatorname{mid}\left(L_{n}\right)\right]\right) \subset M_{n-1} \subset J_{n-2}^{0},
$$

so $\left.g^{2^{n-2}}\right|_{\left[\min L_{n}, \operatorname{mid}\left(L_{n}\right)\right]}$ is linear $\uparrow$ of slope $\lambda_{n} t_{n-2}$. According to Lemma 4.1 (ix) one has $g^{2^{n-2}}\left(\min L_{n}\right)=\min L_{n-1}$. Now (6) and Lemma $4.1($ iii $)+\left(\right.$ iv) imply that $g^{i}\left(\left[\min L_{n}, \operatorname{mid}\left(L_{n}\right)\right]\right) \subset[0,1]$ for all $1 \leq i \leq$ $2^{n-2}$. This is our statement for $k=n$.

- Suppose that the statement is true for $k$ with $3 \leq k \leq n$. By (5) one has $\lambda_{n} \cdots \lambda_{k} \cdot t_{n-2} \cdots t_{k-2}\left|L_{n}\right| / 2 \leq\left|L_{k-1}\right| / 2$ so that

$$
g^{2^{n-2}+2^{n-3}+\cdots+2^{k-2}}\left(\left[\min L_{n}, \operatorname{mid}\left(L_{n}\right)\right]\right) \subset\left[\min L_{k-1}, \operatorname{mid}\left(L_{k-1}\right)\right] .
$$

The map $g$ is of slope $\lambda_{k-1}$ on this interval, $g\left(\min L_{k-1}\right)=\min M_{k-2}$ by Lemma 4.1 (viii), and $g\left(\left[\min L_{k-1}, \operatorname{mid}\left(L_{k-1}\right)\right]\right) \subset M_{k-2}$ by (6). Since $M_{k-2} \subset J_{n-1}^{0}$, the map $g^{2^{n-2}+2^{n-3}+\cdots+2^{k-2}+2^{k-3}}$ is linear $\uparrow$ of slope $\lambda_{n} \ldots \lambda_{k-1} \cdot t_{n-2} \ldots t_{k-3}$ on $\left[\min L_{n}, \operatorname{mid}\left(L_{n}\right)\right]$, and it $\operatorname{maps}$ $\min L_{n}$ to $\min L_{k-2}$ by Lemma $4.1(\mathrm{ix})$. Moreover $g^{i}\left(\left[\min L_{n}, \operatorname{mid}\left(L_{n}\right)\right]\right)$ $\subset[0,1]$ for all $0 \leq i \leq 2^{n-2}+2^{n-3}+\cdots+2^{k-2}+2^{k-3}$ by Lemma 4.1(iv) and the induction hypothesis. This is the statement for $k-1$. 
For $k=2$ we conclude that $g^{2^{n-2}+\cdots+2^{0}}=g^{2^{n-1}-1}$ is linear $\uparrow$ of slope $\prod_{i=2}^{n} \lambda_{i} \prod_{i=0}^{n-2} t_{i}$ on $\left[\min L_{n}, \operatorname{mid}\left(L_{n}\right)\right]$, with $g^{2^{n-1}-1}\left(\min L_{n}\right)=\min L_{1}$ and $g^{2^{n-1}-1}\left(\left[\min L_{n}, \operatorname{mid}\left(L_{n}\right)\right]\right) \subset\left[\min L_{1}, \operatorname{mid}\left(L_{1}\right)\right]$. The map $g$ is of slope $\lambda_{1}$ on this interval, hence, according to the definition of $\lambda_{n}$, assertion (ii) holds for all $n \geq 2$; it also trivially holds for $n=1$. The inductive statement for $k=2$ also shows that

$$
g^{i}\left(\left[\min L_{n}, \operatorname{mid}\left(L_{n}\right)\right]\right) \subset[0,1] \quad \text { for all } 0 \leq i \leq 2^{n-1}-1, n \geq 1 .
$$

Assertions (i) and (ii) and Lemma 4.1(vi) imply (iii).

One has $I_{n}^{1}=\bigcup_{k \geq n+1}\left(I_{k}^{0} \cup L_{k}\right) \cup[a, 1]$. From the definition of $g$ one can see that

$$
\max \left\{g(x) \mid x \in I_{k}^{0} \cup L_{k}\right\}=g\left(\operatorname{mid}\left(L_{k}\right)\right),
$$

and therefore $g\left(I_{k}^{0} \cup L_{k}\right) \subset\left[0, \operatorname{mid}\left(M_{k-1}\right)\right]$ by $(6)$. Hence

$$
g\left(I_{n}^{1}\right) \subset\left[0, \operatorname{mid}\left(M_{n}\right)\right]=J_{n}^{0} \cup\left[\min M_{n}, \operatorname{mid}\left(M_{n}\right)\right],
$$

which is (iv).

By Lemma 4.1(iii) $+($ vii $), g^{2^{n}-1}\left(J_{n}^{0}\right)=I_{n}^{1}$ and

$$
g^{2^{n-1}-1}\left(\left[\min M_{n}, \operatorname{mid}\left(M_{n}\right)\right]\right)=\left[\min L_{n}, \operatorname{mid}\left(L_{n}\right)\right],
$$

and by (ii), $g^{2^{n-1}}\left(\left[\min L_{n}, \operatorname{mid}\left(L_{n}\right)\right]\right)=\left[1,1+x_{n}\right]$. Combining with (iv) we get

$$
g^{2^{n}}\left(I_{n}^{1}\right) \subset I_{n}^{1} \cup\left[1,1+x_{n}\right] .
$$

Moreover $g^{i}\left(J_{n}^{0}\right) \subset[0,1]$ for all $0 \leq i \leq 2^{n}-2$ and $g^{i}\left(\left[\min M_{n}, \operatorname{mid}\left(M_{n}\right)\right]\right) \subset$ $[0,1]$ for all $0 \leq i \leq 2^{n-1}-2$ according to Lemma 4.1(iv). In addition, $g^{2^{n-1}+i-1}\left(\left[\min M_{n}, \operatorname{mid}\left(M_{n}\right)\right]\right)=g^{i}\left(\left[\min L_{n}, \operatorname{mid}\left(L_{n}\right)\right]\right) \subset[0,1]$ for all $0 \leq$ $i \leq 2^{n-1}-1$ by $(7)$. Therefore

$$
g^{i}\left(I_{n}^{1}\right) \subset[0,1] \quad \text { for all } 0 \leq i<2^{n} .
$$

Finally, $g\left(\left[1,1+x_{n}\right]\right)=\left[0, \operatorname{mid}\left(M_{n+1}\right)\right] \subset J_{n}^{0}$, and (i) implies that $g^{2^{n}}\left(\left[1,1+x_{n}\right]\right) \subset I_{n}^{1}$. Combined with (9), (8) and Lemma 4.1(iv), this gives $(\mathrm{v})$.

Now we show that $g$ is continuous at the point $a$, as claimed at the beginning of the section.

Lemma 4.3. The map g defined above is continuous.

Proof. We just have to show the continuity at $a$. It is clear from the definition that $g$ is continuous at $a^{+}$. According to Lemma 4.2(iv) one has $g\left(I_{n}^{1}\right) \subset J_{n-1}^{0}$. This implies that $g$ is continuous at $a^{-}$since $\lim _{n \rightarrow+\infty} \max J_{n}^{0}$ $=0$. 
To end this subsection, let us explain the main underlying ideas of the construction of $g$ by comparing it with the map $f$ built in Section 3. The map $g$ and $f$ are similar on the set $\bigcup_{n \geq 1} I_{n}^{0}$ (which is the core of the dynamics of $f$ ); the only difference is the length of the intervals. For $f$ we showed that $K=\bigcap_{n \geq 0} \bigcup_{i=0}^{2^{n}-1} f^{i}\left(J_{n}^{0}\right)$ has a non-degenerate connected component $B$ and it can be proven that the endpoints of $B$ are $f$-non-separable. The same remains true for $g$ with $B=[a, 1]=\bigcap_{n \geq 0} I_{n}^{1}$ (the fact that $a, 1$ are $g$-nonseparable will be proven in Proposition 4.4). For $f$ we proved that $\partial K \subset$ $\omega(0, f)$, hence $\partial B \subset \omega(0, f)$; for $g$ it is not true that $\{a, 1\} \subset \omega(0, g)$ because the orbit of 0 stays in $[0, a]$. The construction of $g$ on the intervals $L_{n}$ allows one to approach 1 from the outside of $[0,1]$ : we will see in Proposition 4.4 that $\omega\left(1+x_{0}, g\right)$ contains both $a$ and 1 , which implies chaos in the sense of Li-Yorke. On the other hand, the proof showing that $\left.f\right|_{\omega(0, f)}$ is transitive and sensitive fails for $g$ because $\omega(0, g)$ does not contain $\{a, 1\}$ and $\omega\left(1+x_{0}, g\right)$ is not transitive. We will see in Proposition 4.7 that $g$ has no transitive sensitive subsystem at all.

\section{2. $g$ is chaotic in the sense of Li-Yorke}

Proposition 4.4. Let $g$ be the map defined in Section 4.1. Then the set $\omega\left(1+x_{0}, g\right)$ is infinite and contains the points a, 1 , which are $g$-non-separable. Consequently, the map $g$ is chaotic in the sense of Li-Yorke.

Proof. Lemma 4.2(iii) implies that $g^{2^{n+1}}\left(1+x_{n}\right)=1+x_{n+1}$ for all $n \geq 0$. Since $x_{n} \rightarrow 0$ as $n$ goes to infinity, this implies that $1 \in \omega\left(1+x_{0}, g\right)$. Moreover Lemma 4.2(i) implies that $g^{2^{n}}(1)=\min I_{n+1}^{0}=a_{2 n}$ for all $n \geq 1$, hence $a \in \omega(1, g) \subset \omega\left(1+x_{0}, g\right)$.

Suppose $A_{1}, A_{2}$ are two periodic intervals such that $a \in A_{1}$ and $1 \in A_{2}$, and let $p$ be a common multiple of their periods. Since $g(a)=g(1)=0$, it follows that $g^{p}(a)=g^{p}(1) \in A_{1} \cap A_{2}$, and $A_{1}, A_{2}$ are not disjoint. This means that $a, 1$ are $g$-non-separable.

It is well known that a finite $\omega$-limit set is cyclic. Therefore, if $y_{0}, y_{1}$ are two distinct points in a finite $\omega$-set, the degenerate intervals $\left\{y_{0}\right\},\left\{y_{1}\right\}$ are periodic and $y_{0}, y_{1}$ are $g$-separable. This implies that $\omega\left(1+x_{0}, g\right)$ is infinite. We deduce that the map $g$ is chaotic in the sense of Li-Yorke by Theorem 2.2.

4.3. $g$ is not chaotic in the sense of Wiggins. The main result of this subsection is Proposition 4.7 stating that $g$ has no transitive sensitive subsystem. The next lemma is about the location of transitive subsystems.

Lemma 4.5. Let $g$ be the map defined in Section 4.1 and $Y \subset[0,3 / 2]$ a closed invariant subset with no isolated point such that $\left.g\right|_{Y}$ is transitive. Then 
(i) $Y \subset[0, a]$,

(ii) $Y \subset \bigcup_{i=0}^{2^{n}-1} g^{i}\left(J_{n}^{0}\right)$ for all $n \geq 1$,

(iii) $g^{i}\left(J_{n}^{0} \cap Y\right)=g^{i}\left(J_{n}^{0}\right) \cap Y=g^{i \bmod 2^{n}}\left(J_{n}^{0}\right) \cap Y$ for all $i \geq 0, n \geq 0$.

Proof. By transitivity there exists $y_{0} \in Y$ such that $\omega\left(y_{0}, g\right)=Y$; in particular the set $Y^{\prime}=\left\{g^{k}\left(y_{0}\right) \mid k \geq 0\right\}$ is dense in $Y$ and $y \in \omega(y, g)$ for all $y \in Y^{\prime}$.

Let $n \geq 0$. By Lemma 4.2(iii), $g^{2^{n+1}}\left(\left[1,1+x_{n}\right]\right)=I_{n+1}^{1} \cup\left[1,1+x_{n+1}\right]$ and hence Lemma $4.2(\mathrm{v})$ shows that for all integers $k \geq 1, g^{k 2^{n+1}}\left(\left[1,1+x_{n}\right]\right) \subset$ $I_{n+1}^{1} \cup\left[1,1+x_{n+1}\right]$ and $g^{i}\left(\left[1,1+x_{n}\right]\right) \subset[0,1]$ for all $i>2^{n+1}, i \notin 2^{n+1} \mathbb{N}$. This implies that

$$
g^{i}\left(\left(1+x_{n+1}, 1+x_{n}\right]\right) \subset\left[0,1+x_{n+1}\right] \quad \text { for all } i \geq 2^{n+1} .
$$

Consequently, there is no $y \in(1,3 / 2]=\bigcup_{n \geq 0}\left(1+x_{n+1}, 1+x_{n}\right]$ such that $y \in \omega(y, g)$, hence $Y^{\prime} \cap(1,3 / 2]=\emptyset$ and by density $Y \cap(1,3 / 2]=\emptyset$.

Since $g^{2^{n}-1}(0)=a_{2 n}$ by Lemma 4.1(ii)+(iii), the point 0 is not periodic, hence $g^{k}(0) \notin[a, 1]$ for all $k \geq 1$. If $y \in(a, 1)$ then $g(y)=0$ and $g^{k}(y) \notin[a, 1]$ for all $k \geq 1$, which implies that $y \notin \omega(y, g)$. Consequently, $Y \cap(a, 1)=\emptyset$. We find that $Y \subset[0, a] \cup\{1\}$, and $1 \notin Y$ because $Y$ has no isolated point; this gives (i).

Let $n \geq 1$. Since $\min L_{n}=\max I_{n}^{0}$ and $\max L_{n}=\min I_{n+1}^{0}$, it follows that $g\left(\min L_{n}\right)=\max J_{n}^{1}$ and $g\left(\max L_{n}\right)=\min J_{n+1}^{1}$ by Lemma 4.1(i). Moreover $\left.g\right|_{\left[\min L_{n}, \operatorname{mid}\left(L_{n}\right)\right]}$ is $\uparrow$ and $\left.g\right|_{\left[\operatorname{mid}\left(L_{n}\right), \max L_{n}\right]}$ is linear $\downarrow$, so there exists $c_{n}$ in $\left[\operatorname{mid}\left(L_{n}\right), \max L_{n}\right]$ such that $g\left(c_{n}\right)=g\left(\min L_{n}\right)$.

Since $g\left(\left[c_{n}, \max L_{n}\right]\right)=\left[\min J_{n+1}^{1}, \max J_{n}^{1}\right]$ is included in $J_{n-1}^{0}$, the map $\left.g^{2^{n-1}}\right|_{\left[c_{n}, \max L_{n}\right]}$ is linear $\downarrow$ by Lemma 4.1(ii). Moreover $M_{n} \subset g\left(\left[c_{n}, \max L_{n}\right]\right)$, hence $g^{2^{n-1}}\left(\left[c_{n}, \max L_{n}\right]\right)$ contains $L_{n}$ by Lemma 4.1(vii). Consequently, there exists $z_{n} \in\left[c_{n}, \max L_{n}\right]$ such that $g^{2^{n-1}}\left(z_{n}\right)=z_{n}$ (Lemma 2.4) and slope $\left(\left.g^{2^{n-1}}\right|_{\left[c_{n}, \max L_{n}\right]}\right) \leq-2$. Then for every $x \in\left[c_{n}, \max L_{n}\right], x \neq z_{n}$, there exists $k \geq 1$ such that $g^{k 2^{n-1}}(x) \notin\left[c_{n}, \max L_{n}\right]$. By Lemma $4.2(\mathrm{v})$ one has $g^{2^{n-1}}\left(I_{n-1}^{1} \cup\left[1,1+x_{n-1}\right]\right) \subset I_{n-1}^{1} \cup\left[1,1+x_{n-1}\right]$, which implies that

$$
\begin{aligned}
& \forall x \in\left[c_{n}, \max L_{n}\right], x \neq z_{n}, \exists k \geq 1, \\
& g^{k 2^{n-1}}(x) \in I_{n}^{0} \cup\left[\min L_{n}, c_{n}\right] \cup I_{n}^{1} \cup\left[1,1+x_{n-1}\right] .
\end{aligned}
$$

We show by induction on $n$ that

$$
\forall n \geq 0, \quad Y^{\prime} \cap I_{n}^{1} \neq \emptyset .
$$

This is true for $n=0$ because $Y \subset[0,1]=I_{0}^{1}$ by (i). Suppose that there exists $y \in Y^{\prime} \cap I_{n-1}^{1}$. Write $I_{n-1}=I_{n-1}^{1}=I_{n}^{0} \cup L_{n} \cup I_{n}^{1}$; to prove that $Y^{\prime} \cap I_{n}^{1} \neq \emptyset$ we split into four cases. 
- If $y \in I_{n}^{1}$ there is nothing to do.

- If $y \in I_{n}^{0}$ then $g^{2^{n-1}}(y) \in I_{n}^{1}$ by Lemma 4.1(vi) and $g^{2^{n-1}}(y) \in Y^{\prime}$.

- If $y \in\left[\min L_{n}, c_{n}\right]$ then $g(y) \in g\left(\left[\min L_{n}, \operatorname{mid}\left(L_{n}\right)\right]\right.$ and $g^{2^{n-1}}(y) \in$ $\left[1,1+x_{n}\right]$ by Lemma 4.2(ii), which is impossible because $Y \subset[0, a]$ by (i).

- If $y \in\left[c_{n}, \max L_{n}\right]$ then $y \neq z_{n}$ because $Y$ is infinite. In addition $g^{j}(y) \in[0,1]$ for all $j \geq 0$ according to (i). Then (10) says that there exists $j \geq 1$ such that $g^{j}\left(y^{\prime}\right) \in I_{n}^{0} \cup\left[\min L_{n}, c_{n}\right] \cup I_{n}^{1}$ and one of the first three cases applies.

One has $g\left(I_{n}^{1}\right) \subset J_{n}^{0} \cup\left[\min M_{n}, \operatorname{mid}\left(M_{n}\right)\right]$ by Lemma 4.2(iv) and also $g^{2^{n}-1}\left(\left[\min M_{n}, \operatorname{mid}\left(M_{n}\right)\right]\right)=g^{2^{n-1}}\left(\left[\min L_{n}, \operatorname{mid}\left(L_{n}\right)\right]\right)=\left[1,1+x_{n}\right]$ by Lemmas 4.1(vii) and 4.2(ii). Together with (i) this implies that

$$
g\left(Y \cap I_{n}^{1}\right) \subset J_{n}^{0} .
$$

Now (11) and (12) combined with Lemma 4.1(i)+(iii) imply that

$$
Y \subset \bigcup_{i=0}^{2^{n}-1} g^{i}\left(J_{n}^{0}\right) \quad \text { for all } n \geq 1,
$$

which is (ii); furthermore $Y \cap g^{i}\left(J_{n}^{0}\right)=Y \cap g^{i \bmod 2^{n}}\left(J_{n}^{0}\right)$ for all $i \geq 0$. Since $g(Y)=Y$ it is clear that $g^{i}\left(J_{n}^{0} \cap Y\right) \subset g^{i}\left(J_{n}^{0}\right) \cap Y$ and that $g^{2^{n}}\left(g^{i}\left(J_{n}^{0}\right) \cap Y\right) \subset$ $g^{2^{n}+i}\left(J_{n}^{0}\right) \cap Y$; thus

$$
g^{i}\left(J_{n}^{0} \cap Y\right)=g^{i}\left(J_{n}^{0}\right) \cap Y=g^{i \bmod 2^{n}}\left(J_{n}^{0}\right) \cap Y \quad \text { for all } i \geq 0 .
$$

The next lemma is the key tool in the proof of Proposition 4.7. It relies on the knowledge of the precise location of $g^{i}\left(J_{n}^{0}\right)$ in $\bigcup_{1 \leq k \leq n} I_{n}^{0}$.

Lemma 4.6. Let $g$ be the map defined in Section 4.1. For all $n \geq 1$ and all $0 \leq k \leq 2^{n}-1$ one has $\operatorname{slope}\left(\left.g^{2^{n}-1-k}\right|_{g^{k}\left(J_{n}^{0}\right)}\right) \geq 1$.

Proof. A (finite) word $B$ is an element of $\mathbb{N}^{n}$ for some $n \in \mathbb{N}$. If $B, B^{\prime}$ are two words, $B B^{\prime}$ denotes their concatenation and $|B|=n$ is the length of $B$.

We define inductively a sequence $\left(B_{n}\right)_{n \geq 1}$ of words by:

- $B_{1}=1$,

- $B_{n}=n B_{1} B_{2} \ldots B_{n-1}$,

and we define the infinite word $\omega=(\omega(i))_{i \geq 1}$ by concatenating the $B_{n}$ 's: $\omega=$ $B_{1} B_{2} B_{3} \ldots B_{n} \ldots$ A straightforward induction shows that $\left|B_{n}\right|=2^{n-1}$; thus $\left|B_{1}\right|+\left|B_{2}\right|+\cdots+\left|B_{k}\right|=2^{k}-1$ and the word $B_{k+1}$ begins at $\omega\left(2^{k}\right)$, which gives

$$
\begin{gathered}
\omega\left(2^{k}\right)=k+1 \\
\omega\left(2^{k}+1\right) \ldots \omega\left(2^{k+1}-1\right)=B_{1} \ldots B_{k}=\omega(1) \ldots \omega\left(2^{k}-1\right) .
\end{gathered}
$$


We prove by induction on $k \geq 1$ that

$$
g^{i-1}\left(J_{n}^{0}\right) \subset I_{\omega(i)}^{0} \quad \text { for all } n \geq k, 1 \leq i \leq 2^{k}-1 .
$$

- Case $k=1: J_{n}^{0} \subset I_{1}^{0}=I_{\omega(1)}^{0}$ for all $n \geq 1$.

- Suppose that (15) holds for $k$ and let $n \geq k+1$. Since $J_{n}^{0} \subset J_{k+1}^{0}$, Lemma 4.1(iii) implies that $g^{2^{k}-1}\left(J_{n}^{0}\right) \subset I_{k+1}^{0}$, and thus $g^{2^{k}}\left(J_{n}^{0}\right) \subset J_{k}^{0}$ by Lemma 4.1(i). By induction one has $g^{i-1}\left(J_{k}^{0}\right) \subset I_{\omega(i)}^{0}$ for all $1 \leq$ $i \leq 1^{k}-1$, and (14) yields $\omega(i)=\omega\left(2^{k}+i\right)$ for all $1 \leq i \leq 2^{k}-1$. Consequently, $g^{2^{k}+i-1}\left(J_{n}^{0}\right) \subset I_{\omega\left(2^{k}+i\right)}^{0}$ for all $1 \leq i \leq 2^{k}-1$. Together with the induction hypothesis this gives (15) for $k+1$.

Let $\mu_{n}=\operatorname{slope}\left(\left.g\right|_{I_{n}^{0}}\right)$. By definition of $g$ one has

$$
\mu_{n}=\frac{\operatorname{slope}\left(\varphi_{n}\right)}{\prod_{i=1}^{n-1} \operatorname{slope}\left(\varphi_{i}\right)} .
$$

It is straightforward from (15) that for all $2 \leq k \leq 2^{n}-1$,

$$
\operatorname{slope}\left(\left.g^{k-1}\right|_{J_{n}^{0}}\right)=\prod_{i=1}^{k-1} \mu_{\omega(i)} .
$$

By Lemma 4.1(ii)+(iii) the map $\left.g^{2^{n}-1}\right|_{J_{n}^{0}}$ is linear and $g^{2^{n}-1}\left(J_{n}^{0}\right)=I_{n}^{1}$, thus

$$
\operatorname{slope}\left(\left.g^{2^{n}-1}\right|_{J_{n}^{0}}\right)=\frac{\left|I_{n}^{1}\right|}{\left|J_{n}^{0}\right|}=\prod_{i=1}^{n} \frac{1-2 / 3^{i}}{1 / 3^{i}} .
$$

Since slope $\left(\varphi_{i}\right)=\frac{\left|I_{i}^{1}\right|}{\left|I_{i}^{0}\right|}=\frac{1-2 / 3^{i}}{1 / 3^{i}}$, we get

$$
\operatorname{slope}\left(\left.g^{2^{n}-1}\right|_{J_{n}^{0}}\right)=\prod_{i=1}^{2^{n}-1} \mu_{\omega(i)}=\prod_{i=1}^{n} \operatorname{slope}\left(\varphi_{i}\right) .
$$

We show by induction on $n \geq 1$ that for all $1 \leq k \leq 2^{n}-1$,

$$
\prod_{i=1}^{k} \mu_{\omega(i)}=\prod_{i=1}^{n} \operatorname{slope}\left(\varphi_{i}\right)^{\varepsilon_{i}} \quad \text { for some } \varepsilon_{i}=\varepsilon(i, k, n) \in\{0,1\} .
$$

- $\mu_{\omega(1)}=\mu_{1}=\operatorname{slope}\left(\varphi_{1}\right)$; this gives the case $n=1$.

- Suppose that the statement is true for $n$. Then

$$
\begin{aligned}
\prod_{i=1}^{2^{n}} \mu_{\omega(i)} & =\prod_{i=1}^{2^{n}-1} \mu_{\omega(i)} \cdot \mu_{n+1} \\
& =\prod_{i=1}^{n} \operatorname{slope}\left(\varphi_{i}\right) \frac{\operatorname{slope}\left(\varphi_{n+1}\right)}{\prod_{i=1}^{n} \operatorname{slope}\left(\varphi_{i}\right)} \\
& =\operatorname{slope}\left(\varphi_{n+1}\right) .
\end{aligned}
$$

This is (18) for $n+1$ and $k=2^{n}$ with $\varepsilon(i, k, n 1)=0$ for $1 \leq i \leq n$ and $\varepsilon\left(n+1,2^{n}, n+1\right)=1$. 
Next, $\omega\left(2^{n}+1\right) \ldots \omega\left(2^{n+1}-1\right)=\omega(1) \ldots \omega\left(2^{n}-1\right)$ by (14), so if $2^{n}+1 \leq k \leq 2^{n+1}-1$ then

$$
\begin{aligned}
\prod_{i=1}^{k} \mu_{\omega(i)} & =\prod_{i=1}^{2^{n}} \mu_{\omega(i)} \prod_{i=2^{n}+1}^{k} \mu_{\omega(i)}=\operatorname{slope}\left(\varphi_{n+1}\right) \prod_{i=1}^{k-2^{n}} \mu_{\omega(i)} \\
& =\operatorname{slope}\left(\varphi_{n+1}\right) \prod_{i=1}^{n} \operatorname{slope}\left(\varphi_{i}\right)^{\varepsilon\left(i, k_{2}^{n}, n\right)}
\end{aligned}
$$

That is, (18) holds with $\varepsilon(i, k, n+1)=\varepsilon\left(i, k-2^{n}, n\right)$ for all $1 \leq i \leq n$ and $\varepsilon(n+1, k, n+1)=1$. This concludes the induction.

Equations (16) and (18) show that for all $1 \leq k \leq 2^{n}-1$,

$$
\operatorname{slope}\left(\left.g^{k}\right|_{J_{n}}\right)=\prod_{i=1}^{k+1} \mu_{\omega(i)}=\prod_{i=1}^{n} \operatorname{slope}\left(\varphi_{i}\right)^{\varepsilon_{i}} \quad \text { for some } \varepsilon_{i} \in\{0,1\} \text {. }
$$

Since

$$
\operatorname{slope}\left(\left.g^{2^{n}-1-k}\right|_{g^{k}\left(J_{n}^{0}\right)}\right)=\frac{\operatorname{slope}\left(\left.g^{2^{n}-1}\right|_{J_{n}^{0}}\right)}{\operatorname{slope}\left(\left.g^{k}\right|_{J_{n}^{0}}\right)},
$$

(17) and (19) imply that $\operatorname{slope}\left(\left.g^{2^{n}-1-k}\right|_{g^{k}\left(J_{n}\right)}\right)$ is a product of at most $n$ terms of the form slope $\left(\varphi_{i}\right)$. This concludes the proof of the lemma because $\operatorname{slope}\left(\varphi_{i}\right) \geq 1$ for all $i \geq 1$.

Proposition 4.7. The map $g$ defined in Section 4.1 is not Wiggins chaotic.

Proof. Let $Y \subset[0,3 / 2]$ be a closed invariant subset such that $\left.g\right|_{Y}$ is transitive. We assume that $Y$ has no isolated point, otherwise $\left.g\right|_{Y}$ is not sensitive.

The sets $\left(g^{i}\left(J_{n}^{0} \cap Y\right)\right)_{0 \leq i \leq 2^{n}-1}$ are closed and by Lemma $4.1(\mathrm{v})$ they are pairwise disjoint; let $\delta_{n}>0$ be the minimal distance between two of them. If $x, x^{\prime} \in Y$ and $\left|x-x^{\prime}\right|<\delta_{n}$ then there is $0 \leq i \leq 2^{n}-1$ such that $x, x^{\prime} \in g^{i}\left(J_{n}^{0}\right)$ and $g^{k}(x), g^{k}\left(x^{\prime}\right) \in g^{i+k \bmod 2^{n}}\left(J_{n}^{0}\right)$ for all $k \geq 0$ by Lemma 4.5(ii)+(iii). Let

$$
\varepsilon_{n}=\max \left\{\operatorname{diam}\left(g^{i}\left(J_{n}^{0}\right) \cap Y\right) \mid 0 \leq i<2^{n}\right\} .
$$

By Lemma 4.6, $\operatorname{diam}\left(g^{k}\left(J_{n}^{0}\right) \cap Y\right) \leq \operatorname{diam}\left(g^{2^{n}-1}\left(J_{n}^{0}\right) \cap Y\right)$ for all $0 \leq k \leq$ $2^{n}-1$. By Lemma 4.1(iii) one has $g^{2^{n}-1}\left(J_{n}^{0}\right)=I_{n}^{1}$ and by Lemma 4.5(i) one has $I_{n}^{1} \cap Y \subset\left[a_{2 n}, a\right]$; thus $\varepsilon_{n} \leq \operatorname{diam}\left(I_{n}^{1} \cap Y\right) \leq a-a_{2 n}$, which implies that

$$
\lim _{n \rightarrow+\infty} \varepsilon_{n}=0 .
$$

This shows that $\left.g\right|_{Y}$ is not sensitive.

Theorem 1.8 now follows from Propositions 4.4 and 4.7. 


\section{References}

[1] J. Auslander and J. Yorke, Interval maps, factors of maps and chaos, Tohoku Math. J. 32 (1980), 177-188.

[2] M. Barge and J. Martin, Chaos, periodicity, and snakelike continua, Trans. Amer. Math. Soc. 289 (1985), 355-365.

[3] -, -, Dense orbits on the interval, Michigan Math. J. 34 (1987), 3-11.

[4] F. Blanchard, E. Glasner, S. Kolyada, and A. Maass, On Li-Yorke pairs, J. Reine Angew. Math. 547 (2002), 51-68.

[5] L. Block, Homoclinic points of mappings of the interval, Proc. Amer. Math. Soc., 72 (1978), 576-580.

[6] L. S. Block and W. A. Coppel, Dynamics in One Dimension, Lecture Notes in Math. 1513, Springer, 1992.

[7] L. Block, J. Guckenheimer, M. Misiurewicz, and L. S. Young, Periodic points and topological entropy of one dimensional maps, in: Global Theory of Dynamical Systems, Lecture Notes in Math. 819, Springer, 1980, 18-34.

[8] A. M. Blokh, On sensitive mappings of the interval, Uspekhi Mat. Nauk 37 (1982), no. 2, 189-190 (in Russian); English transl.: Russ. Math. Surveys 37 (1982), 203-204.

[9] J.-P. Delahaye, Fonctions admettant des cycles d'ordre n'importe quelle puissance de 2 et aucun autre cycle, C. R. Acad. Sci. Paris Sér. A-B 291 (1980), A323-A325; Addendum, 291 (1980), A671.

[10] R. L. Devaney, An Introduction to Chaotic Dynamical Systems, 2nd ed., AddisonWesley, 1989.

[11] B.-S. Du, Smooth weakly chaotic interval maps with zero topological entropy, in: Dynamical Systems and Related Topics (Nagoya, 1990), Adv. Ser. Dynam. Systems 9, World Sci., River Edge, NJ, 1991, 72-79.

[12] V. V. Fedorenko, A. N. Sarkovskii, and J. Smítal, Characterizations of weakly chaotic maps of the interval, Proc. Amer. Math. Soc. 110 (1990), 141-148.

[13] X.-C. Fu and J. Duan, Infinite-dimensional linear dynamics systems with chaoticity, J. Nonlinear Sci. 9 (1999), 197-211.

[14] K. Janková and J. Smítal, A characterization of chaos, Bull. Austral. Math. Soc. 34 (1986), 283-292.

[15] S. Kolyada and L'. Snoha, Some aspects of topological transitivity - a survey, in: Iteration Theory (ECIT 94, Opava), Karl-Franzens-Univ. Graz, Graz, 1997, 3-35.

[16] S. H. Li, w-chaos and topological entropy, Trans. Amer. Math. Soc. 339 (1993), $243-249$.

[17] T. Y. Li and J. A. Yorke, Period three implies chaos, Amer. Math. Monthly 82 (1975), 985-992.

[18] V. Jiménez López, An explicit description of all scrambled sets of weakly unimodal functions of type $2^{\infty}$, Real Anal. Exchange 21 (1995/96), 664-688.

[19] M. Misiurewicz, Horseshoes for continuous mappings of an interval, in: Dynamical Systems (Bressanone, 1978), Liguori, Napoli, 1980, 125-135.

[20] M. Misiurewicz and J. Smítal, Smooth chaotic maps with zero topological entropy, Ergodic Theory Dynam. Systems 8 (1988), 421-424.

[21] S. Ruette, Chaos for continuous interval maps: a survey of relationship between the various sorts of chaos, http://www.math.u-psud.fr/ ruette/.

[22] O. M. Šarkovskiı̌, Co-existence of cycles of a continuous mapping of the line into itself, Ukrain. Mat. Ž. 16 (1964), 61-71 (in Russian).

[23] J. Smítal, Chaotic functions with zero topological entropy, Trans. Amer. Math. Soc. 297 (1986), 269-282. 
[24] S. Wiggins, Introduction to Applied Nonlinear Dynamical Systems and Chaos, Texts in Appl. Math. 2, Springer, 1990.

[25] J. C. Xiong, A chaotic map with topological entropy [zero], Acta Math. Sci. (English Ed.) 6 (1986), 439-443.

Laboratoire de Mathématiques

Topologie et Dynamique, Bâtiment 425

Université Paris-Sud

F-91405 Orsay Cedex, France

E-mail: Sylvie.Ruette@math.u-psud.fr

Received September 7, 2004

Revised version January 21, 2005 